Portland State University

PDXScholar

$11-2017$

\title{
Ion Transport across Biological Membranes by Carborane-Capped Gold Nanoparticles
}

\author{
Marcin P. Grzelczak \\ University of Liverpool \\ Stephen P. Danks \\ University of Liverpool \\ Robert C. Klipp \\ Portland State University, KlippRob@gmail.com \\ Domagoj Belic \\ University of Liverpool \\ Adnana Zaulet \\ Institut de Ciencia de Materials de Barcelona
}

See next page for additional authors

Follow this and additional works at: https://pdxscholar.library.pdx.edu/phy_fac

Part of the Physics Commons

Let us know how access to this document benefits you.

\section{Citation Details}

Grzelczak, M. P., Danks, S. P., Klipp, R. C., Belic, D., Zaulet, A., Kunstmann-Olsen, C., \& ... Brust, M. (2017). Ion Transport across Biological Membranes by Carborane-Capped Gold Nanoparticles. ACS Nano, 11(12), 12492-12499.

This Article is brought to you for free and open access. It has been accepted for inclusion in Physics Faculty Publications and Presentations by an authorized administrator of PDXScholar. Please contact us if we can make this document more accessible: pdxscholar@pdx.edu. 


\section{Authors}

Marcin P. Grzelczak, Stephen P. Danks, Robert C. Klipp, Domagoj Belic, Adnana Zaulet, Casper KunstmannOlsen, Dan F. Bradley, Tatsuya Tsukuda, Clara Viñas, Francesc Teixidor, Jonathan J. Abramson, and Mathias Brust 


\title{
Ion Transport across Biological Membranes by Carborane-Capped Gold Nanoparticles
}

\author{
Marcin P. Grzelczak, ${ }^{* \dagger}{ }^{\dagger}$ Stephen P. Danks, ${ }^{\dagger}$ Robert C. Klipp, ${ }^{\S}$ Domagoj Belic, ${ }^{\dagger}$ Adnana Zaulet, ${ }^{\dagger}$

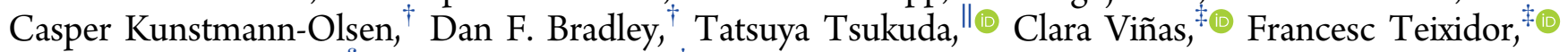 \\ Jonathan J. Abramson, ${ }^{\S}$ and Mathias Brust ${ }^{*}{ }^{\dagger}$ (0) \\ ${ }^{\dagger}$ Department of Chemistry, University of Liverpool, Liverpool L69 7ZD, United Kingdom \\ ${ }^{\ddagger}$ Institut de Ciencia de Materials de Barcelona, ICMAB-CSIC, Campus UAB, E-08193 Bellaterra, Spain \\ ${ }^{\S}$ Physics Department, Portland State University, Portland, Oregon 97207, United States \\ "Department of Chemistry, School of Science, The University of Tokyo, 7-3-1 Hongo, Bunkyo-ku, Tokyo 113-0033, Japan
}

\section{Supporting Information}

ABSTRACT: Carborane-capped gold nanoparticles (Au/carborane NPs, 2-3 nm) can act as artificial ion transporters across biological membranes. The particles themselves are large hydrophobic anions that have the ability to disperse in aqueous media and to partition over both sides of a phospholipid bilayer membrane. Their presence therefore causes a membrane potential that is determined by the relative concentrations of particles on each side of the membrane according to the Nernst equation. The particles tend to adsorb to both sides of the membrane and can flip across if changes in membrane potential require their repartitioning. Such changes can be made either with a potentiostat in an electrochemical cell or by competition with another partitioning ion, for example, potassium in the presence of its specific transporter valinomycin. Carborane-capped gold nanoparticles have a ligand shell full of voids, which stem from the packing of near spherical ligands on a near spherical metal core. These voids are normally filled with sodium or potassium ions, and the charge is overcompensated by excess electrons in the metal core. The anionic particles are therefore able to take up and release a certain payload of cations and to adjust their net charge accordingly. It is demonstrated by potential-dependent fluorescence spectroscopy that polarized phospholipid membranes of vesicles can be depolarized by ion transport mediated by the particles. It is also shown that the particles act as alkali-ion-specific transporters across free-standing membranes under potentiostatic control. Magnesium ions are not transported.

KEYWORDS: artificial ion transporters, gold nanoparticles, carborane, membrane potential, fluorescence spectroscopy, electrochemistry

on transport across biological membranes is a fundamental
phenomenon ubiquitous in nature. ${ }^{1-6}$ Photosynthesis, the
respiratory chain, ATP production, muscle contraction,
neuronal signaling, and many other key biological processes
depend on it. ${ }^{7,8}$ Numerous active and passive transport
mechanisms exist ranging from simple ion channels to highly
complex and regulated energy-converting nanomachineries.
Naturally occurring ion transporters are proteins, whereas
artificial ones can be made in different ways, usually from
peptides, ${ }^{9}$ macrocyclic organic compounds, ${ }^{10,11}$ or appropri-
ately functionalized polymers including DNA. ${ }^{12-15}$ Substances
that affect ion transport or its regulation play important roles in
both basic research and drug development. Carborane-capped
gold nanoparticles ${ }^{16}$ in the $1-4$ nm size range are known for
their ability to store and release cationic charge that is
counterbalanced by excess electrons in the metal core. ${ }^{17}$ This is
possible due to the inevitable voids in the ligand shell, which
are caused by the near spherical shape of the carborane ligand.
It may also be assisted by the dipole moment of the ligand, which in this case is pointing toward the gold surface. Carboranes have the interesting property that the orientation of their dipole moment relative to a surface can be controlled by the position of the two vicinal carbon atoms. ${ }^{18}$ Gold nanoparticles loaded up with cations readily disperse in water as net polyanions but, if possible, tend to associate with water/oil interfaces or with hydrophobic membranes. Unlike most other nanoscopic forms of gold, ${ }^{19}$ the particles are cytotoxic and can enter biological cells by direct membrane penetration and finally lodge inside membranous structures including the mitochondria. Here, we report that $2-3 \mathrm{~nm}$ gold nanoparticles protected by mercaptocarborane ligands (a) readily penetrate

Received: September 16, 2017

Accepted: November 21, 2017

Published: November 21, 2017 
a)

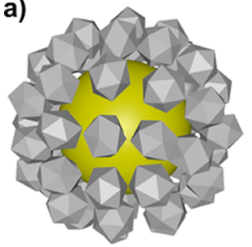

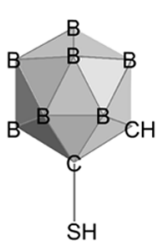

b)

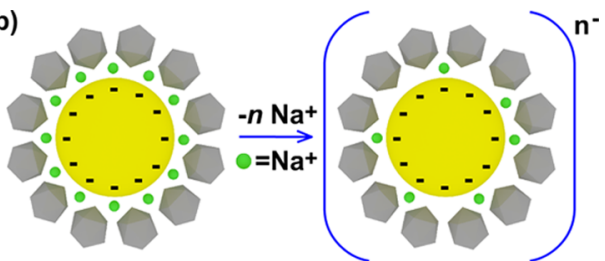

Figure 1. Schematic representation of nanoparticles and charge storage. (a) Carborane-capped gold nanoparticles. (b) Charge storage in metallic core (electrons) and ligand shell (sodium ions) and formation of a water-dispersible polyanion by dissociation of sodium ions from the ligand shell. ${ }^{17}$

a)

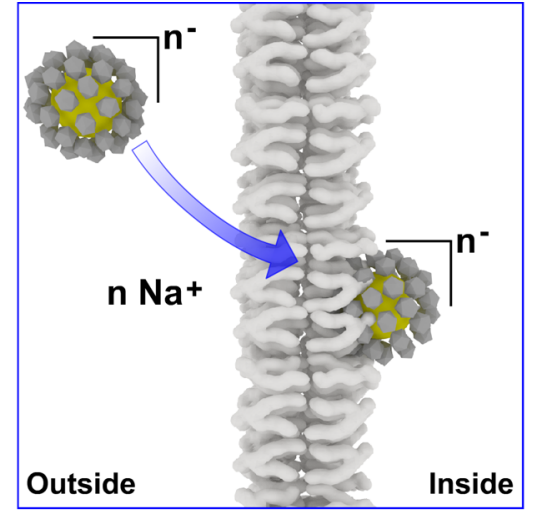

c)

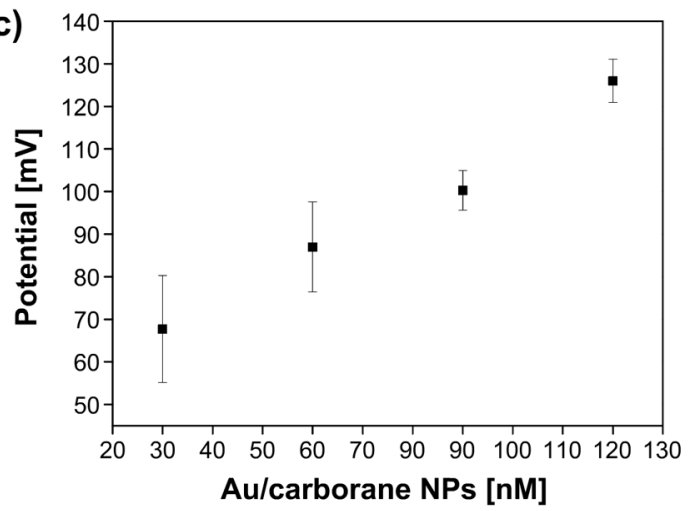

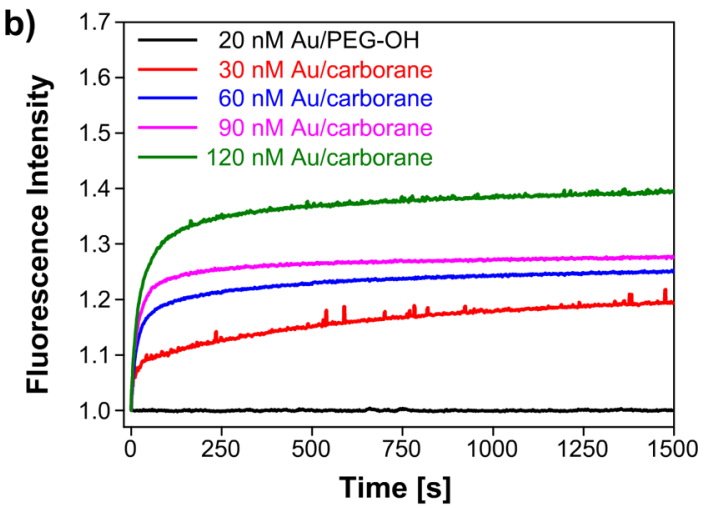

d)

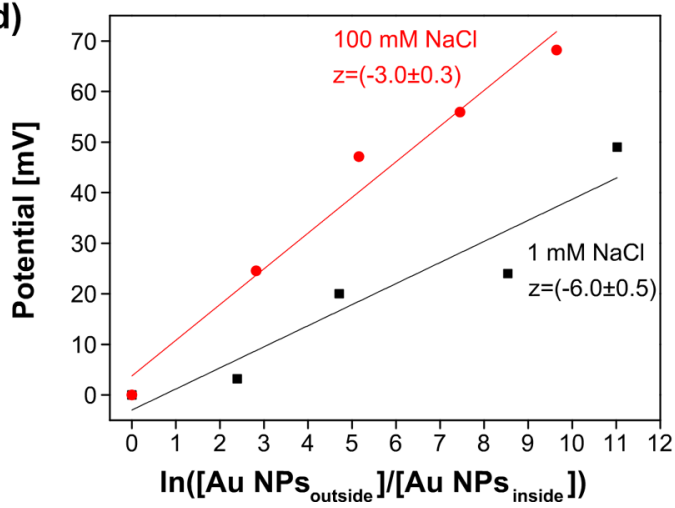

Figure 2. Membrane polarization by Au/carborane NPs. (a) Schematic representation of membrane polarization after addition of anionic Au/ carborane NPs to the aqueous medium outside the vesicles. While the particles readily transfer across the membrane, counterions remain on the outside of the vesicle. (b) Fluorescence response to the addition of Au/carborane NPs in a range of different concentrations in the absence of electrolyte. Note that polyethylene glycol-coated gold nanoparticles (Au/PEG-OH NPs) have no effect. (c) Membrane potential estimated from the data presented in (b). (d) Plot derived from the Nernst equation for two different $\mathrm{NaCl}$ concentrations. The membrane potentials were directly measured using a DIB cell (Figure S4a). Note the dependence of the charge of the Au/carborane NPs on the NaCl concentration, -3 at $100 \mathrm{mM}$ and -6 at $1 \mathrm{mM}$. This is likely to be the case also for other alkali ions that can enter the ligand shell.

phospholipid bilayer membranes and establish a membrane potential as partitioning anions and (b) effect the transport of alkali cations across the membrane and hence represent a class of membrane transporters. Two complementary experimental techniques have been used to study these phenomena: (i) voltage-dependent fluorescence spectroscopy of dispersions of vesicles and (ii) potentiometric and potential step experiments across a free-standing bilayer membrane.

\section{RESULTS AND DISCUSSION}

We have previously reported the unexpected ability of the hydrophobic carborane-capped gold nanoparticles $(2-3 \mathrm{~nm})$ to disperse readily in aqueous media. ${ }^{17}$ This is due to the storage of both electronic and ionic charge in the metallic core and in the ligand shell, respectively, as illustrated in Figure 1. Phase transfer experiments with cationic transfer agents have established the anionic character of the nanoparticles, and ion exchange with lithium followed by $\mathrm{Li}$ nuclear magnetic resonance ( $\left.{ }^{6} \mathrm{Li} \mathrm{NMR}\right)$ spectroscopy has shown that alkali cations close to the metal surface compensate most of the excess electronic charge of the metal core. Removal of most charge by acid treatment renders the particles completely hydrophobic. It is useful to recall these findings when interpreting the results presented here.

Before studying the ability of these nanoparticles to act as ion transporters across biological membranes, it is necessary to consider their own propensity as large anions to partition across the membrane and to establish a membrane potential. For this purpose, we used an established experimental approach to monitor the membrane potential of phospholipid vesicles by fluorescence spectroscopy using the potential indicator dye safranin $\mathrm{O}$ that partitions between the membrane and the outer aqueous phase depending on membrane potential. ${ }^{20,21}$ The 

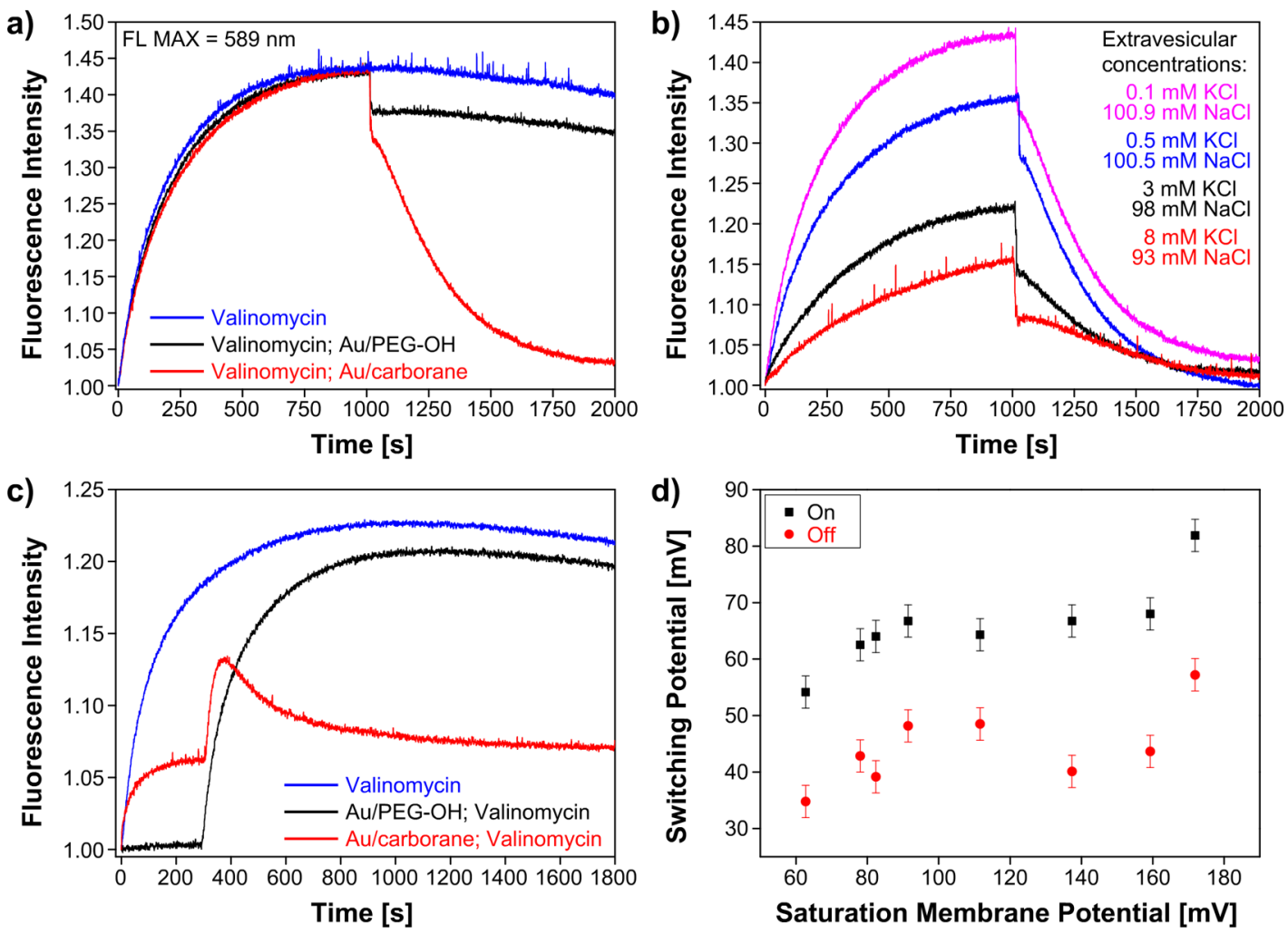

Figure 3. Monitoring the fluorescence of safranin $O$ to probe the polarization and depolarization of the vesicle membrane. (a) All three traces show the initial polarization of the membrane after addition of valinomycin $(11 \mathrm{nM})$ in the presence of safranin $\mathrm{O}(180 \mathrm{nM})$ in the medium outside the vesicles. The concentration of $\mathrm{KCl}$ was $100 \mathrm{mM}$ inside and $0.1 \mathrm{mM}$ outside the vesicles, and that of $\mathrm{NaCl} \mathrm{was} 1 \mathrm{mM}$ inside and $100.9 \mathrm{mM}$ outside, which gives a positive polarity outside. Addition of Au/PEG-OH (20 $\mathrm{nM}$ ) particles (black trace) leads to a rapid small decrease in fluorescence intensity but none attributable to change in membrane potential. This indicates that no charge is transferred by these particles. When instead Au/carborane NPs $(20 \mathrm{nM})$ are added (red trace), besides the familiar small change in signal, the fluorescence decreased exponentially over $600 \mathrm{~s}$. This is attributed to influx of sodium and efflux of potassium ions mediated by Au/carborane NPs and valinomycin. (b) Same as the red trace in (a) but with different potassium ions gradients, i.e., different saturation potentials. Depolarization of the membrane to a final value by Au/carborane NPs occurs over a wide range of potentials. (c) Red trace shows the same experiment as in (a), but the NPs were added first followed by addition of valinomycin $(300 \mathrm{~s})$. After the initial small polarization caused by NPs themselves, the membrane is polarized further upon addition of valinomycin. Note that after further polarization to an onset overpotential depolarization occurs as in (a) and (b). (d) Overpotentials for the onset of depolarization (black) and final potentials after depolarization (red) as a function of saturation membrane potential given by the potassium ion gradient (Figure S5).

fluorescence intensity significantly increases when the dye molecules reside inside the membrane. Vesicles of 100-200 nm diameter with a positive $\zeta$-potential (Table S1) were prepared following the method reported by Stupp and co-workers ${ }^{22}$ and characterized by dynamic light scattering (DLS) and cryotransmission electron microscopy (cryo-TEM) (Figure S1). For calibration purposes, their membrane potential was controlled by carefully adjusting the concentrations of $\mathrm{K}^{+}$ions inside and outside the vesicles in the presence of the $\mathrm{K}^{+}$-specific transporter valinomycin. The fluorescence intensity of safranin $O$ was calibrated against the membrane potential values calculated from the Nernst equation (Figure S2). ${ }^{20,23}$ If, instead of valinomycin, the nanoparticles are added, a rapid polarization of the membrane in the same direction (inside negative) is also observed, but the final value does not depend on the potassium concentrations on both sides of the membrane but only on the amount of nanoparticles added.

This effect is most pronounced in the absence of any added electrolyte other than the particles themselves (Figure $2 b, c$ ). If electrolytes are present, the polarizing effect of the $\mathrm{Au} /$ carborane NPs differs somewhat for different cations (Figure S3). We attribute this polarization to the anionic nanoparticles' ability to transfer across the membrane and, in the absence of cation transport, to determine the membrane potential as the only partitioning ion. They are self-transporting partitioning ions. To quantify this in terms of a Nernst equilibrium, it is necessary to control the concentrations of nanoparticles on both sides of the membrane, which is not possible with the vesicle system but can be achieved using a droplet interface bilayer (DIB) separating two independently accessible electrolyte solutions, as elegantly demonstrated by Bayley et al. (Figure S4a). ${ }^{24-26}$ The results are presented in Figure $2 \mathrm{~d}$. Although these direct measurements of membrane potential in this system are experimentally challenging and hence somewhat noisy, the results are robust and fully commensurate with the predictions from the Nernst equation. Also, the average charge per particle can now be inferred as -6 in $1 \mathrm{mM}$ sodium chloride and -3 in $100 \mathrm{mM}$ sodium chloride. At this stage, there is no evidence that the particles are capable of transporting ions across the membrane other than themselves.

A different scenario presents itself when the membrane is first polarized by the presence of a potassium gradient using the potassium-specific transporter valinomycin (less than 2 molecules per vesicle). Figure $3 a, b$ show how the membrane potential is built up and reaches its saturation value. If then the gold nanoparticles are added ( 1 to 2 particles per vesicle), an 
a)

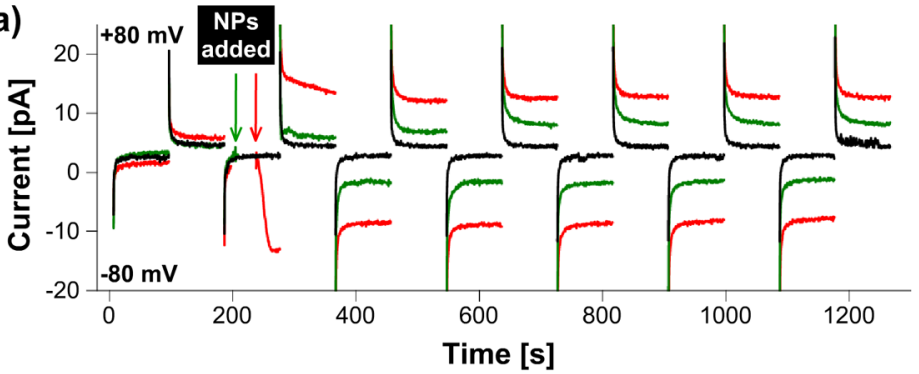

$\mathrm{Ag}|\mathrm{AgCl}| 100 \mathrm{mM} \mathrm{NaCl}, 1 \mathrm{mM} \mathrm{KCl}$ || $100 \mathrm{mM} \mathrm{KCl}, 1 \mathrm{mM} \mathrm{NaCl}|\mathrm{AgCl}| \mathrm{Ag}$ $\mathrm{Ag}|\mathrm{AgCl}| 100 \mathrm{mM} \mathrm{NaCl}|| 100 \mathrm{mM} \mathrm{NaCl}|\mathrm{AgCl}| \mathrm{Ag}$ $\mathrm{Ag}$ | $\mathrm{AgCl}|100 \mathrm{mM} \mathrm{NaCl}, 1 \mathrm{mM} \mathrm{KCl} \mathrm{||} 100 \mathrm{mM} \mathrm{KCl}, 1 \mathrm{mM} \mathrm{NaCl}| \mathrm{AgCl} \mid \mathrm{Ag}$ Control without NPs

c)

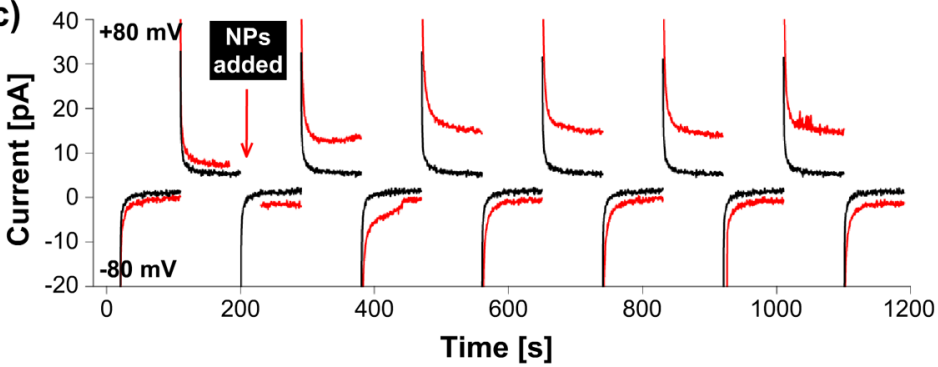

$\mathrm{Ag}|\mathrm{AgCl}| 100 \mathrm{mM} \mathrm{NaCl}, 0.666 \mathrm{mM} \mathrm{MgCl}_{2}|| 66.66 \mathrm{mM} \mathrm{MgCl}_{2}, 1 \mathrm{mM} \mathrm{NaCl}|\mathrm{AgCl}| \mathrm{Ag}$ $\mathrm{Ag}|\mathrm{AgCl}| 100 \mathrm{mM} \mathrm{NaCl}, 0.666 \mathrm{mM} \mathrm{MgCl}_{2}|| 66.66 \mathrm{mM} \mathrm{MgCl}_{2}, 1 \mathrm{mM} \mathrm{NaCl}|\mathrm{AgCl}| \mathrm{Ag}$ b)

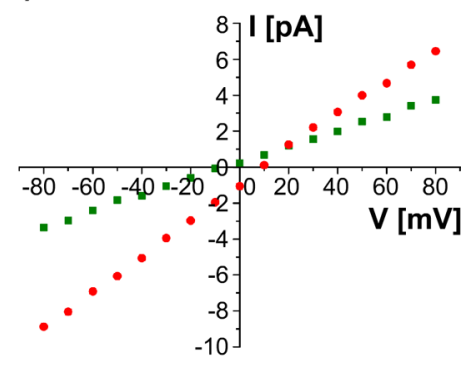

d)

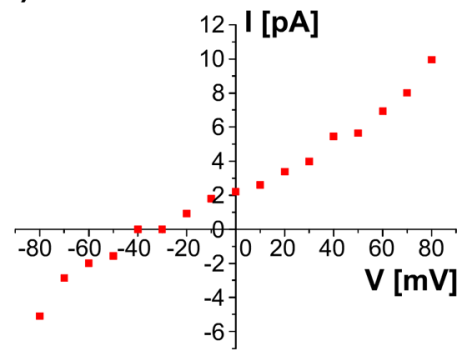

Control without NPs

Figure 4. Current-voltage responses in potential step experiments on free-standing phospholipid bilayer membranes. (a) Current traces in response to potential steps of $-\mathbf{8 0} /+\mathbf{8 0} \mathrm{mV}$. The electrochemical cells are color coded underneath. Note that the largest currents are obtained in the presence of steep gradients of potassium and sodium concentrations across the membrane. (b) $I-V$ curves corresponding to (a). Each point represents the steady-state current of the potential step experiment. The graph has been corrected to eliminate a small offset current (3-4 pA) that is present even in the absence of NPs. The intercept of $+8 \mathrm{mV}$ shows that $\mathrm{K}^{+}$is preferentially transported over $\mathrm{Na}^{+}$. (c,d) Same as (a) and (b) but using sodium and magnesium instead. The intercept at $-40 \mathrm{mV}$ shows that sodium is transported preferentially to magnesium. This selectivity also indicates that simple defect formation in the membrane can be excluded as a transport mechanism.

initial fast but small decline in fluorescence intensity is always observed. We interpret this as an artifact perhaps due to quenching and/or reabsorption of light by the metallic particle.

Then, the fluorescence intensity decreases, gradually approaching a constant final value that quite accurately corresponds to the membrane potential the particles alone would have caused. The effective canceling of the potential initially determined by the potassium gradient is explained by the influx of $\mathrm{Na}^{+}$and the efflux of $\mathrm{K}^{+}$ions both from high to low concentration, indicating that the gold nanoparticles added under these conditions do provide a mechanism for $\mathrm{Na}^{+}$ transport across the membrane as well as for $\mathrm{K}^{+}$, in addition to valinomycin. As there are now transporters for sodium and potassium present in the membrane, both ions can freely flow in and out until their concentrations inside and outside the vesicles are equal and they cause the membrane potential to drop to zero (really to the small value determined by the partitioning of the nanoparticles). For a discussion of transport rates see Supporting Information (Index 7). While the nanoparticles were not found to transport ions that polarize the membrane, this experiment shows unequivocally that they support ion transport to depolarize the membrane in the presence of a competing polarizing mechanism. For comparison, if instead, our standard $\mathrm{Au} / \mathrm{PEG}-\mathrm{OH}$ nanoparticles of the same size are added, only the initial fast drop in signal intensity is observed but no subsequent depolarization of the membrane. ${ }^{27}$ An unexpected phenomenon is revealed by an experiment identical to that shown in (Figure $3 a, b$ ) except that the nanoparticles are now added before the addition of valinomycin rather than afterward. The result is shown in Figure 3c. Upon addition of the particles, as expected, a small membrane potential is generated (30-40 $\mathrm{mV}$ inside negative) due to the partitioning of the anionic particles. When then valinomycin is added, the membrane is further polarized, making the inside more negative due to the increased $\mathrm{K}^{+}$ selectivity of the membrane induced by valinomycin. This initially confirms that the nanoparticles do not transport ions under these conditions, until the membrane potential reaches a value of $20-30 \mathrm{mV}$ more negative inside than the potential caused by the particles alone. At this point, the membrane suddenly begins to depolarize gradually to the original value determined by the nanoparticles. We suggest that this empirical overpotential for depolarization is needed to activate the repartitioning of nanoparticles if they have prepolarized the membrane by having been added to one side only. After this initial polarization, the anionic particles will reside predominantly on the inside of the vesicles and will first have to cross the membrane before they can support the influx of sodium ions. The observed overpotential is always between 20 and 30 $\mathrm{mV}$ negative of the equilibrium potential caused by the particles alone. We believe that this would disappear if we had an equal concentration of particles on both sides of the membrane, which in the present system is experimentally not possible. A further shortcoming of this experimental approach is that we can only measure membrane potentials with an outside positive/inside negative polarity as it was not possible to 

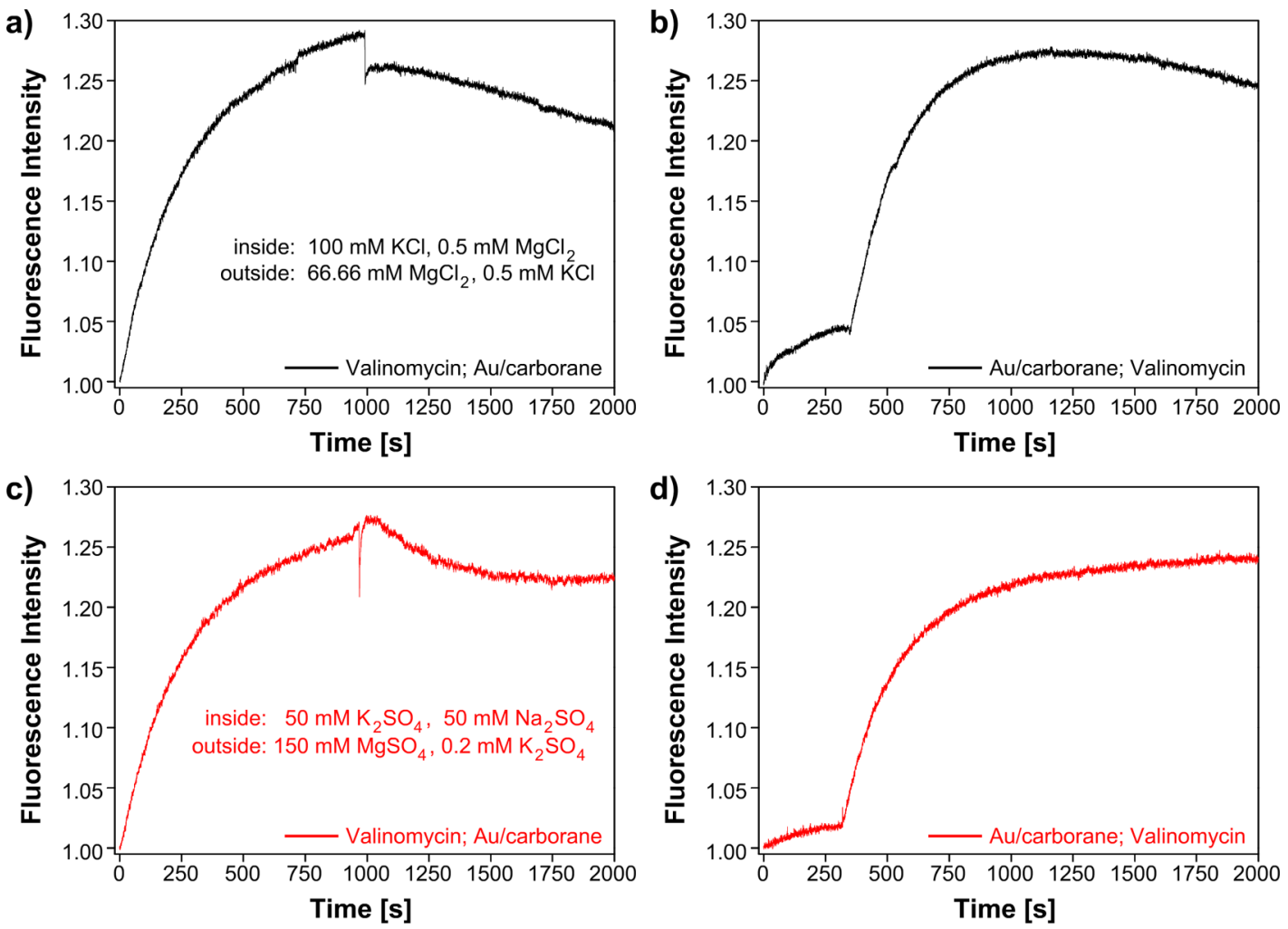

Figure 5. (a) Polarization with potassium gradient after addition of valinomycin. Addition of Au/carborane NPs does not lead to depolarization if magnesium is chosen as the partitioning ion. (b) Same as (a) but the Au/carborane NPs were added before addition of valinomycin at $300 \mathrm{~s}$. Again no depolarization occurs. (c) Polarization with potassium gradient after addition of valinomycin and attempt to further polarize the membrane using Au/carborane NPs as a transporter and sodium as partitioning ion. (d) Same as (c) but the NPs were added before of addition of valinomycin. All electrolyte concentrations are given in the insets.

prepare the vesicles with the potential sensitive dye incorporated inside, which would have been necessary to measure membrane potentials of opposite polarity. Also, ionic currents across the membranes are inferred but are not measured directly. To overcome these limitations and to validate our findings independently by an alternative method, we used planar bilayer membranes separated by two compartments of a small 3D-printed electrochemical cell (Figure S4b). ${ }^{28,29}$ These are conceptually similar to the DIB that have been used to measure the membrane potential caused by the partitioning of the nanoparticles. The current responses to alternating potential steps of up to $-80 /+80 \mathrm{mV}$ applied across the membrane were recorded under various conditions. The results are shown in Figure 4. While sodium and potassium are both transported readily with a small preference for potassium, significant differences are found when comparing sodium and magnesium (Figure $4 c, d$ ). The intercept of $-40 \mathrm{mV}$ in Figure $4 \mathrm{~d}$ indicates a clear preference for sodium over magnesium. This selectivity has also been observed by electrospray ionization time-of-flight (ESI-TOF) mass spectrometry after ion exchange experiments (Figure S6).

The information provided by both approaches is consistent. The vesicle experiments have established that the nanoparticles act as cation transporters when they depolarize the membrane. Importantly, all attempts failed to polarize the membrane with partitioning ions that use the nanoparticles as transporter. Only the nanoparticles themselves can polarize the membrane regardless of the presence and concentration differences of other ions in the system. The depolarization of a prepolarized membrane is not possible if sodium is replaced by magnesium
(Figure 5a,b), confirming the very low affinity of the particles for magnesium established by the electrochemical study. Further polarization of a prepolarized membrane is also not possible (Figure $5 \mathrm{c}, \mathrm{d}$ ), further confirming that the particles only transport ions if the resulting flow depolarizes the membrane. In this sense, the particles behave differently from a transporter such as valinomycin, which can be used to polarize the membrane with a potassium gradient. The particles alone will not do this; instead they always establish a membrane potential that is due to their own partitioning as anions.

The inability to polarize the membrane by the transport of cations in the presence of a concentration gradient strongly suggests that the particles do not simply open channels through which ions can freely flow across the membrane. A shuttle mechanism is more likely by which the particles fill up with cations on the side of high concentration, flip across the membrane, and release them on the side of low concentration, probably becoming more negative upon cation release unless the empty vacancies are immediately filled up with other cations. Only if the cations are released on the negatively polarized side of the membrane (depolarization) can the membrane potential push back the negatively charged particle to the positive side, where it is replenished with cations and the process repeats. Our current understanding of this process is summarized in Figure 6. Release of cations on the positive side of the membrane (polarization) would stop the process as the particle would remain trapped on that side of the membrane. The vesicle experiments also revealed that membrane depolarization requires an overpotential which we attribute to having the particles only added to one side of the membrane. 


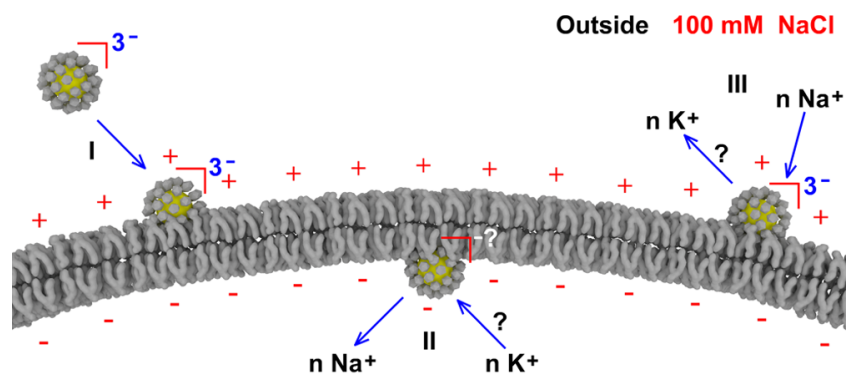

Inside $100 \mathrm{mM} \mathrm{KCl}$

Figure 6. Scheme of membrane depolarization by transport of sodium and potassium ions. (I) Particles are added to the sodium rich dispersion of vesicles and adsorb to the vesicle membrane (Figure S8). Note, the membrane has been polarized (outside positive) by the presence of a potassium ion concentration gradient and the potassium specific carrier valinomycin (not shown). (II) As the particles penetrate the membrane, sodium ions are released inside the vesicle (down their concentration gradient). (III) As long as particles reside within the membrane, they can shuttle sodium ions across by passive transport down their concentration gradient. In parallel with the mechanism provided by valinomycin, the particles could also contribute to the export of potassium. The process stops when the concentrations of sodium and potassium inside and outside the vesicles are equal. The remaining potential of the depolarized membrane is then due to the partitioning of the anionic nanoparticles.

Indeed, the electrochemical experiments do not show this overpotential and further seem to suggest that the particles act as transporters much like valinomycin (Figure S7). This is because by the very nature of these experiments, under potentiostatic control, the membrane is always polarized in such a way that cations cross the membrane from the positive to the negative side. The unusual property of the particles not to support a polarizing flow of cations would thus not have been noticed by the electrochemical investigation alone.

\section{CONCLUSION}

In conclusion, we have demonstrated by two complementary experimental approaches that cation transport across phospholipid membranes can be facilitated by ligand-capped gold nanoparticles, which by themselves are large partitioning anions that form a Nernst equilibrium and result in the buildup of a membrane potential. Electrochemical experiments have established little selectivity within the alkali ions tested but high selectivity of alkali over alkaline earth ions. A condition for the particles' activity as transporters is that the membrane potential is kept away from the equilibrium potential determined by the particles. This is done by either providing a chemical potential difference and an ion selective pathway or by controlling the membrane potential directly with a potentiostat. In the absence of both, the particles do not act as transporters for any ions but themselves and cannot be used, for example, like valinomycin, to polarize a membrane with a suitable partitioning ion.

Our findings establish functionalized nanoparticles as a class of ion transporters with some interesting properties. The phenomena observed suggest a simple model of a nanoscale electrostatically driven shuttle. The presence of the metallic core should, in parallel, enable electronic conduction which would create interesting opportunities for the use of biological membranes. The design of nano- and microscale systems with coupled electronic and ionic transport between compartments separated by a membrane is the subject of ongoing research in our laboratories.

\section{METHODS}

Chemicals. Hydrogen tetrachloroaurate(III) hydrate $\left(\mathrm{HAuCl}_{4}\right.$ $\left.3 \mathrm{H}_{2} \mathrm{O}\right)$ and sodium borohydride $\left(\mathrm{NaBH}_{4}\right)$ were supplied by Aldrich. Both cationic liposome kit and asolectin from soybean were purchased from Sigma. HS- $\mathrm{C}_{11}-\mathrm{PEG}_{4}-\mathrm{OH}$ was supplied by ProChimia. The cationic exchange resin, strongly acidic PA with a total exchange capacity of 2.0 mequiv/ $\mathrm{mL}$, and a water content of $46-52 \%$, loaded with the desired cation chloride was provided by Panreac. Mercaptocarborane, 1-SH-1,2-closo- $\mathrm{C}_{2} \mathrm{~B}_{10} \mathrm{H}_{11}$, was synthesized according to the literature. ${ }^{30,31}$ All salts were purchased from Sigma. Synthetic grade methanol, ethanol, and Milli-Q-grade (MQ) water were used in all preparations.

Unilamellar Vesicles. A $10 \mathrm{mM}$ stock solution of phospholipids was made by dilution of lyophilized powder from a liposome kit (cholesterol $9 \mu \mathrm{mol}$, L- $\alpha$-phosphatidylcholine $63 \mu \mathrm{mol}$, stearylamine $18 \mu \mathrm{mol}$ ) in chloroform. ${ }^{22,23}$ One milliliter aliquots of the stock solution were rotary evaporated to dryness and for a further $2 \mathrm{~h}$ in order to remove all residues of organic solvent. MQ water or $\mathrm{KCl}$, $\mathrm{Na}_{2} \mathrm{SO}_{4}, \mathrm{~K}_{2} \mathrm{SO}_{4}$ buffer solutions were used to hydrate the phospholipids depending on the requirements of each experiment (agitate overnight). In order to make the suspension of phospholipids more homogeneous, it was heated to $60{ }^{\circ} \mathrm{C}$ followed by $6-9$ freezethaw cycles using liquid nitrogen. Next, the homogeneous phospholipid solution was extruded 20 times through a mini-extruder (Avanti) with a $100 \mathrm{~nm}$ pore-size polycarbonate filter (Whatman) to form vesicles of about $100 \mathrm{~nm}$ diameters. Purification of such prepared vesicles and buffer exchange were carried out by using dialysis tubing MWCO 12000-14000 (Serva).

Preparation of Ligand-Capped 2-3 nm Gold Nanoclusters. Both $\mathrm{Au} / \mathrm{PEG}-\mathrm{OH}$ and $\mathrm{Au} /$ carborane gold nanoclusters were synthesized following a literature method. ${ }^{17}$ All the reaction components were mixed in the following final concentrations: capping agents [HS- $\left.\mathrm{C}_{11}-\mathrm{PEG}_{4}-\mathrm{OH}\right]$ or [mercaptocarborane] $=3 \mathrm{mM}$, $\left[\mathrm{HAuCl}_{4}\right]=3 \mathrm{mM},\left[\mathrm{NaBH}_{4}\right]=9.5 \mathrm{mM}$. In the case of mercaptocarborane-capped gold nanoclusters solvent was rota evaporated followed by washing of excess capping agent molecules with diethyl ether. In the final step, dark-brown residue was dissolved in isopropyl alcohol and filtered to remove the remaining sodium borohydride and other insoluble contaminants. PEGylated gold nanoclusters were washed by using ultra-high-speed centrifuge at $163000 \mathrm{rcf}$ for $1 \mathrm{~h}$, and the pellet was redispersed in a 9:1 mixture of water and ethanol. In total, the purification process was repeated three times where final product was redispersed in MQ water only. All particles were additionally characterized by ultraviolet-visible spectroscopy (UV-vis), high-resolution transmission electron microscopy (HR-TEM), and Fourier transform infrared spectroscopy (FTIR) (Figures S9 and S10).

Membrane Potential Changes Monitored by Fluorescence Spectroscopy. The suspension of vesicles with a final concentration of phospholipids of $1 \mathrm{mM}$ was placed in a fluorimetric cuvette followed by addition of the potential probe dye safranin $\mathrm{O}(180 \mathrm{nM}$ final concentration). Then the fluorescence intensity was allowed to equilibrate for $2 \mathrm{~min}$. The kinetic mode was used to detect continuous fluorescence intensity changes at $589 \mathrm{~nm}$ with an excitation wavelength of $521 \mathrm{~nm}$. In membrane depolarization experiments, concentrations varying from 11 to $15 \mathrm{nM}$ valinomycin, and $20 \mathrm{nM} \mathrm{Au}$ / carborane NPs were used. The fluorescence intensity was monitored over $2000 \mathrm{~s}$. Experiments including $\mathrm{NaCl}, \mathrm{KCl}$, and $\mathrm{MgCl}_{2}$ salts were performed at a $\mathrm{pH}$ range of 6.8-6.9. Experiment including sulfates (Figure $5 \mathrm{c}, \mathrm{d}$ ) was carried out in similar $\mathrm{pH}$ values $6.8-6.9$ using 10 $\mathrm{mM}$ sodium phosphate buffer.

Membrane Formation in the Droplet Interface Bilayer Cell. The chamber of a 3D-printed polyacrylamide cell (Figure S4a) was filled with aqueous electrolyte $(300 \mu \mathrm{L})$, and then a lipid-containing decane solution $(150 \mu \mathrm{L})$ was added on the top, so that a lipid monolayer formed at the interface. A droplet of electrolyte $(\sim 3 \mu \mathrm{L})$ 
was pipetted onto an agarose-coated $\mathrm{Ag} / \mathrm{AgCl}$ electrode and maneuvered, using a micromanipulator, so that the droplet was in the lipid-decane solution, where a monolayer of lipids surrounded the droplet. It was then moved to be in contact with the interface monolayer, where a bilayer membrane formed between the two aqueous phases. The formation of the membranes was tracked by using electrochemical methods. The average value of capacitance of membranes created using this procedure was $16 \pm 2 \mathrm{nF}$, and resistance was in the range of $10^{8}-10^{9} \Omega$. In each experiment, the membrane was formed with equal electrolyte and $\mathrm{Au} /$ carborane NP concentration in both aqueous phases called "in" and "out" (Figure S4a). Thereafter, gradual addition (via micropipette) of $\mathrm{Au} /$ carborane NPs to underlying planar aqueous medium (out) built up the concentration gradient of NPs across the membrane.

Formation of the Supported Planar Bilayer Membranes. Two $\mathrm{Ag} / \mathrm{AgCl}$ electrodes were each fixed into one of two compartments of a 3D-printed polyacrylamide cell, with an aperture of $\sim 270 \mu \mathrm{m}$ diameter separating the two compartments (Figure S4b). Electrolyte solution $(200 \mu \mathrm{L})$ was added into each compartment to cover the electrodes, but not enough for the aqueous phase to reach the aperture. Fifty microliters of phospholipid solution made of asolectin from soybean mixture dissolved in decane $(25 \mathrm{mg} / \mathrm{mL})$ was added on top of the aqueous phase and left for $20 \mathrm{~min}$ for monolayers to form at the interface between the aqueous and organic phases. The monolayers were brought into contact by raising the level of the aqueous phase, via alternating micropipette additions of electrolyte into each compartment, until the aperture was fully submerged. The formation of the membrane was monitored by cyclic voltammetry using a Metrohm $\mu$ Autolab III potentiostat and confirmed via capacitance and resistance measurements. In a standard experiment, capacitance of the formed membrane was in the range of $80-170 \mathrm{pF}$, yielding average specific capacitance value of $0.248 \pm 0.06 \mu \mathrm{F} / \mathrm{cm}^{2}$. Specific resistance of such formed membranes was in the range of $10^{7}-10^{8} \Omega \cdot \mathrm{cm}^{2}{ }^{32}$ In each experiment, either NPs or valinomycin were added to both sides cis and trans of phospholipid bilayer membrane, reaching their final concentration of $200 \mathrm{nM}$. All experiments were carried out at a $\mathrm{pH}$ range of 7.1-7.2 using $20 \mathrm{mM}$ MOPS buffer (3( $N$-morpholino)propanesulfonic acid).

$\zeta$-Potential and DLS Measurements. The $\zeta$-potential measurements were performed on the earlier purified nanoparticles and vesicle solutions. Three measurements, each including 10 runs, were used to estimate average $\zeta$-potential value. Malvern Zetasizer Nano ZS Zen3600 was used for both $\zeta$-potential and DLS measurements.

Infrared. Colloidal suspensions of gold nanoparticles were deposited on IR windows by using a drop-casting method. Aqueous solutions of $\mathrm{Au} / \mathrm{PEG}-\mathrm{OH}$ were drop-casted on the $\mathrm{ZnSe}$ and $\mathrm{Au} /$ carborane NPs suspended in ethanol on the $\mathrm{CaF}_{2} \mathrm{IR}$ windows. Powder of pure mercaptocarborane ligand was characterized by using attenuated total reflection (ATR) mode.

HR-TEM. The Au NP samples were prepared by drop-casting $3 \mu \mathrm{L}$ of Au NP colloidal dispersion onto a holey carbon film, 300 mesh copper TEM grid (Agar Scientific), which was then dried in air for an hour before insertion into the microscope. HR-TEM was performed on a JEOL JEM 2100FCs equipped with a spherical aberration corrector (CEOS $\mathrm{GmbH}$ ), operating at an accelerating voltage of 200 $\mathrm{kV}$.

Cryo-TEM. Holey carbon film, 300 mesh, or Quantifoil R2/2, 200 mesh copper TEM grids (Agar Scientific), was glow-discharged in a Quorum 150T S turbo-pumped glow-discharge system. The samples were prepared by mixing the lipid vesicle solution with $50 \mathrm{nM}$ colloidal dispersion of Au NPs. Three microliters of the prepared solution was drop-casted onto a glow-discharged TEM grid placed inside an FEI Vitrobot Mk2 vitrification system that was set to $8{ }^{\circ} \mathrm{C}$ and relative humidity of $98 \%$. The samples were blotted $2 \times 2$ s before being plunged into liquid ethane. Vitrified samples were transferred onto a Gatan 626 cryogenic sample holder and imaged on an FEI Tecnai Spirit G2 BioTWIN TEM operating at an accelerating voltage of 120 $\mathrm{kV}$, using an Olympus-SIS MegaView III digital camera. Cryo-TEM imaging was performed in low-dose mode (electron beam current density of $6.3 e^{-} / \AA^{2} \mathrm{~s}$, with the total electron dose per imaged area of $\left.<50 e^{-} / \AA^{2}\right)$ at a defocus ranging from -0.5 to $-1.5 \mu \mathrm{m}$. During cryoTEM, the sample holder temperature was maintained below $-179{ }^{\circ} \mathrm{C}$.

The acquired electron microscopy images were processed using ImageJ 1.46r software (NIH).

Ion Exchange and MALDI-TOF MS. To replace the sodium counterbalancing ions by $\mathrm{Li}^{+}$, approximately $100 \mathrm{mg}$ of prepared sodium-loaded $\mathrm{Au} /$ carborane NPs were dissolved in water. Then, the solution was passed repeatedly through a lithium ion exchange column and subsequently analyzed by ${ }^{7} \mathrm{Li}$ NMR spectroscopy. ${ }^{17}$ Moreover, we interchanged the initial $\mathrm{Na}^{+}$cation with $\mathrm{K}^{+}, \mathrm{Mg}^{2+}$, and $\mathrm{Ca}^{2+}$, by passing a solution of water-soluble sodium reached $\mathrm{Au} /$ carborane nanoparticles through the cation-exchange resin previously loaded with the corresponding cation $\left(\mathrm{K}^{+}, \mathrm{Mg}^{2+}\right.$, and $\left.\mathrm{Ca}^{2+}\right)$. ESI-TOF mass spectra of the ethanol solutions of the $\mathrm{Au} /$ carborane NPs after the ion exchange were measured in the positive-ion mode using a home-built mass spectrometer. ${ }^{33}$ Results showed that $\mathrm{Na}^{+}$can be interchanged only by other alkali metals such as $\mathrm{Li}^{+}$or $\mathrm{K}^{+}$. With alkali-earth metals, such as $\mathrm{Mg}^{2+}$ and $\mathrm{Ca}^{2+}$, there is no cation exchange even if the aqueous solution of the $\mathrm{Au} /$ carborane is passed more than 7 times through the resin as represented in (Figure S6).

\section{ASSOCIATED CONTENT}

Supporting Information

The Supporting Information is available free of charge on the ACS Publications website at DOI: 10.1021/acsnano.7b06600.

Table 1 and Figures S1-S10 as referred to in the text (PDF)

\section{AUTHOR INFORMATION}

\section{Corresponding Authors}

*E-mail: m.grzelczak@liverpool.ac.uk.

*E-mail: mbrust@liverpool.ac.uk.

ORCID

Marcin P. Grzelczak: 0000-0002-3215-1044

Tatsuya Tsukuda: 0000-0002-0190-6379

Clara Viñas: 0000-0001-5000-0277

Francesc Teixidor: 0000-0002-3010-2417

Mathias Brust: 0000-0001-6301-7123

\section{Notes}

The authors declare no competing financial interest.

\section{ACKNOWLEDGMENTS}

The research leading to these results has received funding from the European Research Council under the European Union's Seventh Framework Programme (FP7/2007-2013)/ERC-Advanced Grant Project 321172 PANDORA. We would like to thank $\mathrm{T}$. Watanabe for carrying out the mass spectrometry experiments.

\section{REFERENCES}

(1) Hamill, O. P.; Marty, A.; Neher, E.; Sakmann, B.; Sigworth, F. J. Improved Patch-Clamp Techniques for High-Resolution Current Recording from Cells and Cell-Free Membrane Patches. Pfluegers Arch. 1981, 391, 85-100.

(2) Neher, E.; Sakmann, B. Single-Channel Currents Recorded from Membrane of Denervated Frog Muscle Fibres. Nature 1976, 260, 799-802.

(3) Doyle, D. A.; Morais Cabral, J.; Pfuetzner, R. A.; Kuo, A.; Gulbis, J. M.; Cohen, S. L.; Chait, B. T.; MacKinnon, R. The Structure of the Potassium Channel: Molecular Basis of $\mathrm{K}+$ Conduction and Selectivity. Science 1998, 280, 69-77.

(4) Dutzler, R.; Campbell, E. B.; Cadene, M.; Chait, B. T.; MacKinnon, R. X-Ray Structure of a ClC Chloride Channel at $3.0 \AA$ 
Reveals the Molecular Basis of Anion Selectivity. Nature 2002, 415, 287-294.

(5) Yeagle, P. L. Membrane Transport. In The Membranes of Cells, 3rd ed.; Yeagle, P. L., Ed.; Academic Press: Boston, MA, 2016; pp 335-378.

(6) Stein, W. D.; Litman, T. Carrier-Mediated Transport: Facilitated Disfusion. In Channels, Carriers, and Pumps: An Introduction to Membrane Transport, 2nd ed.; Stein, W. D., Litman, T., Eds.; Elsevier: London, 2015; pp 131-178.

(7) Davies, M. Concept Underlie Models. In Functions of Biological Membranes; Chapman \& Hall: London, 1973; pp 27-54.

(8) Petty, H. R. Bioenergetics: Putting Membranes to Work. In Molecular Biology of Membranes: Structure and Function; Springer: New York, 1993; pp 123-188.

(9) Ghadiri, M. R.; Granja, J. R.; Buehler, L. K. Artificial Transmembrane Ion Channels from Self-Assembling Peptide Nanotubes. Nature 1994, 369, 301-304.

(10) Fyles, T. M.; Loock, D.; Zhou, X. A Voltage-Gated Ion Channel Based on a Bis-Macrocyclic Bolaamphiphile. J. Am. Chem. Soc. 1998, 120, 2997-3003.

(11) Fyles, T. M. Synthetic Ion Channels in Bilayer Membranes. Chem. Soc. Rev. 2007, 36, 335-347.

(12) Langecker, M.; Arnaut, V.; Martin, T. G.; List, J.; Renner, S.; Mayer, M.; Dietz, H.; Simmel, F. C. Synthetic Lipid Membrane Channels Formed by Designed DNA Nanostructures. Science 2012, 338, 932-936.

(13) Burns, J. R.; Seifert, A.; Fertig, N.; Howorka, S. A Biomimetic DNA-Based Channel for the Ligand-Controlled Transport of Charged Molecular Cargo across a Biological Membrane. Nat. Nanotechnol. 2016, 11, 152-156.

(14) Howorka, S. Building Membrane Nanopores. Nat. Nanotechnol. 2017, 12, 619-630.

(15) Maingi, V.; Burns, J. R.; Uusitalo, J. J.; Howorka, S.; Marrink, S. J.; Sansom, M. S. P. Stability and Dynamics of Membrane-Spanning DNA Nanopores. Nat. Commun. 2017, 8, 14784-14796.

(16) Baše, T.; Bastl, Z.; Plzák, Z.; Grygar, T.; Plešek, J.; Carr, M. J.; Malina, V.; Šubrt, J.; Boháček, J.; Večerníková, E.; Křriž, O. Carboranethiol-Modified Gold Surfaces. A Study and Comparison of Modified Cluster and Flat Surfaces. Langmuir 2005, 21, 7776-7785.

(17) Cioran, A. M.; Musteti, A. D.; Teixidor, F.; Krpetić, Ž.; Prior, I. A.; He, Q.; Kiely, C. J.; Brust, M.; Viñas, C. MercaptocarboraneCapped Gold Nanoparticles: Electron Pools and Ion Traps with Switchable Hydrophilicity. J. Am. Chem. Soc. 2012, 134, 212-221.

(18) Schwartz, J. J.; Mendoza, A. M.; Wattanatorn, N.; Zhao, Y.; Nguyen, V. T.; Spokoyny, A. M.; Mirkin, C. A.; Baše, T.; Weiss, P. S. Surface Dipole Control of Liquid Crystal Alignment. J. Am. Chem. Soc. 2016, 138, 5957-5967.

(19) Tonga, G. Y.; Saha, K.; Rotello, V. M. 25th Anniversary Article: Interfacing Nanoparticles and Biology: New Strategies for Biomedicine. Adv. Mater. 2014, 26, 359-370.

(20) Woolley, G.; Kapral, M.; Deber, C. Potential-Sensitive Membrane Association of a Fluorescent Dye. FEBS Lett. 1987, 224, 337-342.

(21) Si, W.; Li, Z. T.; Hou, J. L. Voltage-Driven Reversible Insertion into and Leaving from a Lipid Bilayer: Tuning Transmembrane Transport of Artificial Channels. Angew. Chem., Int. Ed. 2014, 53, $4578-4581$.

(22) Newcomb, C. J.; Sur, S.; Ortony, J. H.; Lee, O.-S.; Matson, J. B.; Boekhoven, J.; Yu, J. M.; Schatz, G. C.; Stupp, S. I. Cell Death versus Cell Survival Instructed by Supramolecular Cohesion of Nanostructures. Nat. Commun. 2014, 5, 3321.

(23) Grzelczak, M. P.; Hill, A. P.; Belic, D.; Bradley, D. F.; Kunstmann-Olsen, C.; Brust, M. Design of Artificial Membrane Transporters from Gold Nanoparticles with Controllable Hydrophobicity. Faraday Discuss. 2016, 191, 495-510.

(24) Tsofina, L. M.; Liberman, E. A.; Babakov, A. V. Production of Bimolecular Protein-Lipid Membranes in Aqueous Solution. Nature 1966, 212, 681-683.
(25) Holden, M. A.; Needham, D.; Bayley, H. Functional Bionetworks from Nanoliter Water Droplets. J. Am. Chem. Soc. 2007, 129, 8650-8655.

(26) Bayley, H.; Cronin, B.; Heron, A.; Holden, M. A.; Hwang, W. L.; Syeda, R; Thompson, J.; Wallace, M. Droplet Interface Bilayers. Mol. BioSyst. 2008, 4, 1191-1208.

(27) Gordillo, G. J.; Krpetić, Z.; Brust, M. Interactions of Gold Nanoparticles with a Phospholipid Monolayer Membrane on Mercury. ACS Nano 2014, 8, 6074-6080.

(28) Montal, M.; Mueller, P. Formation of Bimolecular Membranes from Lipid Monolayers and a Study of Their Electrical Properties. Proc. Natl. Acad. Sci. U. S. A. 1972, 69, 3561-3566.

(29) Montal, M. [50] Formation of Bimolecular Membranes from Lipid Monolayers. Methods Enzymol. 1974, 32, 545-554.

(30) Smith, H. D.; Obenland, C. O.; Papetti, S. A New Series of Organoboranes. IX. The Preparation and Some Reactions of SulfurCarborane Derivatives. Inorg. Chem. 1966, 5, 1013-1015.

(31) Viñas, C.; Benakki, R.; Teixidor, F.; Casabo, J. Dimethoxyethane as a Solvent for the Synthesis of C-Monosubstituted O-Carborane Derivatives. Inorg. Chem. 1995, 34, 3844-3845.

(32) Puchkov, M. N.; Vassarais, R. A.; Korepanova, E. A.; Osipov, A. N. Cytochrome $c$ Produces Pores in Cardiolipin-Containing Planar Bilayer Lipid Membranes in the Presence of Hydrogen Peroxide. Biochim. Biophys. Acta, Biomembr. 2013, 1828, 208-212.

(33) Negishi, Y.; Nobusada, K.; Tsukuda, T. Glutathione-Protected Gold Clusters Revisited: Bridging the Gap between Gold(I)-Thiolate Complexes and Thiolate-Protected Gold Nanocrystals. J. Am. Chem. Soc. 2005, 127, 5261-5270. 